\title{
DIVERSIDAD FLORÍSTICA, VEGETACIONAL Y DE HÁBITATS EN EL ARCHIPIÉLAGO DE LOS CHONOS (REGIÓN DE AISÉN, CHILE)
}

\author{
DIVERSITY OF THE FLORA, VEGETATION AND HABITATS \\ IN THE CHONOS ARCHIPELAGO (AISEN REGION, CHILE)
}

Miguel Álvarez¹, Cristina San Martín², Carla Novoa², Gisela Toledo ${ }^{3} \&$ Carlos Ramírez $^{2}$

\begin{abstract}
RESUMEN
Se compararon la flora y la vegetación de las islas Guamblin, Ipún, Stokes y Kent pertenecientes a la Reserva Nacional "Las Guaitecas" del Archipiélago de Los Chonos en la Patagonia insular chilena. Estas son islas deshabitadas, expuestas al Pacífico y a los vientos del oeste, cubiertas de bosques siempreverdes con depresiones turbosas esfagnosas con Sphagnum magellanicum y turberas pulvinadas con Donatia fascicularis en los montes. Debido a hundimientos o levantamientos de terreno durante los sismos del año 1960, algunas presentan playas de arena con variados hábitats además de plataformas rocosas colonizadas por arbustos. Las diferencias florísticas entre ellas están condicionadas tanto por el sustrato rocoso subyacente como por su relieve.
\end{abstract}

Palabras clave: Similitud florística, vegetación, islas, Patagonia Chilena

\section{ABSTRACT}

We compared the flora and vegetation of the islands Guamblin, Ipún, Stokes and Kent belonging to the National Reserve "Las Guaitecas" in the Chonos Archipelago (Chilean Patagonia). These islands are inhabited and exposed to the Pacific and the West winds, covered with evergreen forest, Sphagnum magellanicum-bogs in depressions and cushion bogs with Donatia fascicularis in the highlands. Due to subsidence or ground surveys during the earthquakes of 1960, some of them have sandy beaches with several habitats and rocky plateaus colonized by shrubs. The floristic differences between the islands are principally determined by their differences on the type of bed-rock and their relief.

Key words: Floristic similitude, vegetation, island, Chilean Patagonia

\footnotetext{
${ }^{1}$ Abteilung Geobotanik und Naturschutz, INRES, Universität Bonn, Alemania malvarez@uni-bonn.de "Vegetation Ecology Group"

${ }^{2}$ Instituto de Botánica, Universidad Austral de Chile, Casilla 567, Valdivia, Chile

${ }^{3}$ Corporación Nacional Forestal (CONAF), Puerto Montt, Chile
} 


\section{INTRODUCCIÓN}

Al sur de la isla grande de Chiloé y hasta la península de Taitao se ubica un extenso archipiélago denominado "Las Guaitecas" en su extremo norte y, "Los Chonos" en el resto (Reiche 1934). El nombre Los Chonos proviene del pueblo aborigen que vivía en ese archipiélago, emparentados con los Alacalufes, eran canoeros nómades que vivían de la pesca y de la caza de lobos marinos (Mena 1985, Manríquez 2004). Los Chonos alcanzaban en sus correrías hasta la isla grande de Chiloé, llegando incluso al canal de Chacao (Rojas 2002). Ocasionalmente, utilizaban la vegetación, ya sea como combustibles o como material para construir sus toldos, comían el fruto del calafate (Berberis buxifolia) y el de los árboles del género Nothofagus (Ñirres y Coihues), como ellos consideraban a ciertas agallas de insectos y cuerpos fructíferos de hongos patógenos del género Cyttaria. También recolectaban papas silvestres (Solanum maglia) que crecían junto a arroyos litorales en las dunas (Ugent et al. 1987). Por último, para los Chonos, el perejil del campo (Apium australe) tenía importancia medicinal (Castro \& Romo 2006).

Este pueblo primitivo fue exterminado por los colonizadores y diezmado por las enfermedades de los blancos, y desde entonces la mayoría de sus islas están prácticamente deshabitadas (Mena 1985). En la actualidad sólo son visitadas ocasionalmente por algueros que colectan "Luga-Luga" (Mazzaella laminarioides), cipreceros que cortan el ciprés de las Guaitecas (Pilgerodendron uviferum) y loberos, que asolan las colonias del lobo fino austral (Arctocephalus australis) para matar sus crías (Castro \& Romo 2006). Algunas de estas actividades son ilegales y por ello, fiscalizadas por la Corporación Nacional Forestal (CONAF). No obstante los anterior, los visitantes esporádicos de las islas que viven en improvisados campamentos de madera y plástico, suelen destruir la vegetación por tala directa o por quemas provocadas por descuido, como lo observara Darwin ya en el siglo XIX (Darwin 1845).

A este interesante y desconocido archipiélago se realizó en enero del año 2004 una expedición científica que contó con el patrocinio de la Dirección de Extensión de la Universidad Austral de Chile (UACh) y de la CONAF. La empresa portuaria NAVIMAG facilitó el traslado de los expedicionarios y sus pertrechos desde Puerto Montt a Puerto Chacabuco. El recorrido por las islas se realizó en la lancha "Petrel" de propiedad de la CONAF visitando 4 islas del archipiélago: Guamblin, Ipún, Stokes y Kent. El grupo de científicos estaba compuesto por 8 estudiantes de la Universidad Austral dedicados a colectar muestras e información relativas a las áreas de Biología Marina, Zoología (Ornitología y Entomología), Micología y Botánica. De esta expedición han resultado dos publicaciones zoológicas (Valenzuela \& Grau 2005, Schrödl \& Grau 2006) y cuatro publicaciones botánicas (Ramírez et al. 2004, 2006a, 2006b, 2007). En estas cuatro últimas publicaciones se analizó por separado la flora y la vegetación de cada una de las islas estudiadas. Sin embargo, hasta el momento no se ha hecho una comparación de los resultados entre las islas usando la totalidad de los datos colectados en la expedición.

El objetivo de este trabajo es hacer una comparación entre las cuatro islas prospectadas en el archipiélago de Los Chonos para determinar el grado de similitud o disimilitud florística entre ellas. Además se discute la correspondencia de las unidades vegetacionales muestreadas con comunidades descritas en la literatura.

\section{Lugar de trabajo}

Las islas Guamblin, Ipún, Stokes y Kent se encuentran en el archipiélago de Los Chonos (Fig. 1) y pertenecen a la Reserva Nacional Las Guaitecas. Además la isla Guamblin fue incorporada al Sistema Nacional de Áreas Silvestres Protegidas por el Estado de Chile (SNASPE) por Decreto $\mathrm{N}^{\circ} 321$ del Ministerio de Agricultura de fecha 1 de julio de 1976, en la categoría de parque con el nombre de Parque Nacional Isla Guamblin (Roqueferre 1987). Todas estas islas fueron afectadas de diferentes maneras por los sismos de mayo de 1960, algunas se levantaron y otras se hundieron incluso de forma asimétrica siendo diferente el nivel alcanzado en distintos lados (Plafker 1972, Fuenzalida 1978). Estas transformaciones geológicas que han alterado el litoral, dificultan la accesibilidad desde el mar por embancamiento o simplemente por la desaparición de los puertos (Fuenzalida 1978, Roqueferre 1987).

El sustrato geológico del archipiélago de Los Chonos corresponde al Complejo Metamórfico Los Chonos. Sobre estos esquistos micáceos descansa una roca sedimentaria formada en el Precámbrico. Una excepción a ello la constituyen las islas Guam- 
blin e Ipún, cuyos sustratos rocosos corresponden a sedimentos terciarios (Peralta \& Homann 1978, Hervé \& Fanning 2001). La mayor superficie de las islas está ocupada por bosques, mientras que en las depresiones o en zonas más húmedas y de suelos más delgados se presentan turberas (Villagra et al. 2009). En los contornos suelen presentarse dunas. Diferencias en el relieve entre estas islas implican diferentes distribuciones de cuerpos de agua y de montañas.

La isla Guamblin es la más grande con un superficie de 15.912 ha y una altitud máxima 250 m s.n.m. Es una meseta muy expuesta, cubierta de bosques con pocos cursos de agua que se presentan principalmente en el litoral. Presenta sólo 3 lagunas en la parte sur. Casi no existen turberas en ella. Las playas con dunas están muy desarrolladas y cubren prácticamente todo el contorno de la isla, lo que tiene que ver con un elevamiento de ella en los sismos de mayo de 1960. En estas dunas existe una gran variedad de hábitats que incluyen pequeñas albuferas, arroyos, pantanos y marismas además de la típica vegetación psamófila.

La isla Ipún alcanza una superficie de 8.418 ha con una altitud máxima de $150 \mathrm{~m}$ s.n.m. Tiene una extensa red de arroyos y arroyuelos que desembocan en el oeste. Además existe una laguna grande llamada Chabranal en el norte y una pequeña llamada laguna Enrique, en el sur. También presenta playas y dunas de arena, que ofrecen variedad de hábitats. Al igual que Guamblin está cubierta de bosques perennifolios interrumpidos por pequeñas turberas esfagnosas. La presencia de un matorral de calafate indica la presencia de un antiguo bosque de coihue, seguramente destruido.

La isla Stokes tiene una superficie de 11.135 ha con una altura máxima de 700 m s.n.m. en la cordillera de su parte sur. Posee una gran cantidad de lagunas (más de 30) en tierras bajas turbosas que cubren un $60 \%$ de la isla, el resto está cubierto de bosques siempreverdes (Villagra et al. 2009). En el monte Philipps y a mayor altura se presentan turberas pulvinadas. Hay algunos cursos de agua, pero casi total ausencia de dunas, al parecer por hundimientos de la costa durante los sismos de mayo de 1960. Este relieve entrega poca diversidad de hábitats.

La isla Kent tiene 9.000 ha de superficie y está separada en tres partes por el estero Las Barrancas que penetra a ella haciendo una $V$. Estas tres partes son abruptas con altitudes que llegan hasta $600 \mathrm{~m}$ s.n.m. en el monte Renán. Hay dos lagunas grandes pero escasa presencia de cursos de agua. La mitad de la isla está cubierta por depresiones turbosas y casi no existen playas salvo en la ensenada Río Coche, lo que indicaría un hundimiento en los años 60. Por lo anterior esta isla tiene también poca diversidad de hábitats. En la única playa se encontró el arbusto Hebe elliptica, una especie que se supone ha llegado por transporte marino desde Nueva Zelandia (Wardle et al. 2001).

El clima templado-frío, oceánico y muy lluvioso es del tipo Cfb en la clasificación de Köppen (Scherhag 1966, Pisano 1981). Amigo \& Ramírez (1998) lo clasifican como bioclima mesotemperado del tipo ultraperhúmedo. Se estima que la precipitación promedio anual debe exceder los $3.500 \mathrm{~mm}$, con temperaturas medias de poca variación entre 7 y $9^{\circ}$ C. Una aproximación al clima de la región se puede observar en el diagrama ombrotérmico de Melinka, estación climatológica ubicada en la isla Ascensión, en el extremo nor-oriental del archipiélago (Fig. 2). En ese lugar la temperatura media anual bordea los $10^{\circ} \mathrm{C}$, con una media de máxima de $13,3^{\circ} \mathrm{C}$ y una media mínima de $6,9^{\circ} \mathrm{C}$. La precipitación alcanza a 3.138 mm promedio anual (Hajek \& di Castri 1975). Pese a que las precipitaciones descienden en verano, se presenta un superávit pluviométrico durante todo el año. Las islas más expuestas presentan árboles y arbustos con forma de "bandera" y sus copas peinadas en forma pareja, por el efecto desecante de los persistentes vientos provenientes del Pacífico. Por efecto del viento también se producen grandes varazones de algas marinas en las playas donde se pudren lentamente (Westermeier \& Ramírez 1979). Las condiciones climáticas varían de acuerdo a la posición y elevación de las islas.

\section{MATERIAL Y MÉTODOS}

Entre enero y febrero de 2004 se visitaron estas islas para tomar las muestras y datos. En ellas se hicieron recorridos tratando de abarcar la máxima extensión y el máximo número de formaciones vegetales posibles. Las unidades de paisaje denominadas formaciones vegetales fueron diferenciadas por su fisionomía. Como los puntos de muestreo se ubicaron dentro de un área de vegetación definida en función a su fisionomía y composición florística, estas unidades fisionómicas y florísticas fueron 
denominadas "comunidades", concepto usado para unidades sintaxonómicas o fitosociológicas de rango no definido (Knapp 1984). De manera que las comunidades corresponden a subunidades de las formaciones diferenciadas por su composición florística. En torno a estos puntos se hicieron listas completas de las especies alli presentes, sin delimitar parcelas de muestreo (plotless sampling). Además se colectó ejemplares de herbario que posteriormente fueron depositados en el Herbario de la Universidad Austral de Chile (VALD). Posteriormente y cuando fue posible, estas comunidades se asimilaron a asociaciones vegetales ya descritas.

Debido a diferencias en el esfuerzo de muestreo en las distintas islas es probable que los resultados de este trabajo contengan un sesgo, sin embargo, tras analizar algunas fotos satelitales estimamos que la gran mayoría de las comunidades presentes en estas islas fue prospectada en este trabajo.

Para la determinación de las especies se usó literatura ad-hoc (e.g. Reiche 1896, 1911, Marticorena \& Rodríguez 1995, 2003) y se comparó los ejemplares con la colección del Herbario VALD. La clasificación utilizada sigue a Heywood (Heywood 1982) y por ello, la clase Dicotiledóneas está tratada en su contexto tradicional, sin diferenciar entre Magnoliopsida y Rosopsida. Además se colectó información acerca del origen geográfico de las especies, reduciéndolo a nativo y adventivo según Marticorena \& Quezada (1985), y las formas de vida según Ellenberg \& Mueller-Dombois (1966). El origen de las especies se usó para comparar la proporción de especies adventicias en las distintas comunidades, mientras que con las formas de vida se confeccionó espectros biológicos para cada formación (Dierschke 1994). Además, se probaron modelos de regresión lineal simple para comparar el número de especies en cada isla versus la superficie, el esfuerzo de muestreo y el número de comunidades muestreadas en ellas, el número de especies adventicias en las comunidades versus su número total de especies y la similitud florística de las islas, usando el índice de Jaccard (1912) versus su distancia geográfica. Finalmente se comparó la composición florística de las comunidades con asociaciones descritas en la literatura usando como criterio la presencia de especies características y la correspondencia ecológica (Dierschke 1994).

El índice de similitud de Jaccard (Sáiz 1980) fue utilizado para la ordenación multivariante de las comunidades mediante el método no métrico de escalamiento multidimensional (non-metric multidimensional scaling). Para los análisis estadísticos se usó el programa $\mathrm{R}$ ( $R$ Development Core Team 2005), incluyendo los paquetes vegan (Oksanen et al. 2009) y simba (Jurasinski 2007), mientras que para la edición de una tabla fitosociológica se usó el programa JUICE (Tichý 2002).

\section{RESULTADOS Y DISCUSIÓN}

\section{Biodiversidad florística}

En las 4 islas estudiadas se encontraron 205 especies vegetales, repartidas en 6 Clases. Las clases de mayor representación fueron las Dicotiledóneas con 121 especies y las Monocotiledóneas con 50 especies (Tabla 1). Los helechos (Polypodiopsida) presentaron 29 especies y las coníferas (Pinopsida) sólo 3. Lycopodiopsida está representada por sólo una especie (Lycopodium gayanum). En el caso de los musgos (clase Bryopsida) sólo se registró a Sphagnum magellanicum por su abundancia en las turberas, mientras que del resto de las especies, un número considerable, mayoritariamente epífitas, se colectó muestras de herbario, las cuales aún no han sido determinadas. Una lista detallada de todas las especies de la flora vascular se presenta en el anexo 1.

Las islas Ipún y Guamblin presentaron 133 $(64,88 \%)$ y $126(61,46 \%)$ especies, mientras que Stokes y Kent alcanzaron sólo a 89 (43,41 \%) y 77 (37,56 \%) especies, respectivamente (Tabla 2). Estos valores muestran una débil correlación con el tamaño de las islas, pero una correlación más alta con el esfuerzo de muestreo y con el número de comunidades muestreadas (Tabla 3). Este último muestra la importancia de la diversidad de ambientes en la determinación de la riqueza florística de las islas más que la superficie en sí. Sin duda que una exploración más completa de estas islas puede arrojar resultados definitivos al respecto.

Participación de las especies alóctonas en la flora De las 205 especies colectadas, 173 (84,39 $\%)$ son nativas y sólo 32 (15,61 \%) son introducidas. El bajo porcentaje de especies introducidas indica un estado de pristinidad aceptable para un área protegida; sin embargo, la presencia de malezas, señala una 
incipiente actividad antrópica especialmente en las islas más expuestas, donde se concentra (Hauenstein et al. 1988). Efectivamente, en las floras de Ipún y Guamblin se encontraron las mayores cantidad de neófitos, 23 y 20 especies, mientras que las islas Stokes y Kent sólo presentaron 4 y 5 especies, respectivamente (Tabla 2). Las especies introducidas se ubican mayoritariamente en dunas, marismas y pantanos costeros (San Martín et al. 1992). Considerando cada comunidad vegetal muestreada existe una débil correlación entre el número de especies adventicias y el número total de especies (Fig. 3), lo cual reafirma el hecho de que la introducción de especies afecta a aquellas comunidades más expuestas a la acción humana, vale decir, aquellas distribuídas cercanas a las costas.

Las especies introducidas son en su mayoría hierbas y sólo una es leñosa (Salix viminalis). Casi todas corresponden a malezas comunes en el continente, sin embargo, llama la atención la presencia de Lycopus europaeus, especie europea, asilvestrada en los pantanos de la región de Los Ríos, presente en las islas Guamblin e Ipún, las más expuestas, y que sólo puede haber llegado allí mediante transporte a través del mar, seguramente con las corrientes litorales que se dirigen al sur (Ramírez \& Romero 1978). Las malezas presentes en las islas, tales como Cirsium vulgare, Galium aparine, Solanum nigrum, Sonchus asper, Sonchus oleraceus y Urtica dioica son indicadoras de nitrógeno (Ramírez et al. 1991), lo que se relaciona con la acumulación de basura orgánica, dejada en las playas y sus cercanías por visitantes esporádicos. En la isla Guamblin se colectó Hydrocotyle ranunculoides, maleza acuática de origen europeo que indica contaminación orgánica en arroyos y lagunas (San Martín et al. 2003). En el caso de Potentilla anserina probablemente se trate de un arqueófito, vale decir, de una especie venida desde Europa en tiempos prehistóricos (Ramírez \& San Martín 1993). En las dunas son comunes las gramíneas introducidas Ammophila arenaria (presente en Guamblin e Ipún) y Leymus arenarius (que sólo falta en Ipún), especies usadas comúnmente para fijar dunas y que se han propagado espontáneamente a lo largo de las costas de Chile (San Martín et al. 1992).

\section{Espectro biológico}

El espectro biológico está dominado por espe- cies hemicriptófitas (Tabla 4) que son hierbas rastreras o en roseta, cuyas yemas de renuevo se ubican a nivel del suelo. A ellas les siguen en abundancia las fanerófitas, especies leñosas de hábito arbóreo o arbustivo. Junto a ellas las plantas trepadoras, epífitas y parasíticas corresponden a las formas de vida típicas del macroclima lluvioso templado dominante en las islas del archipiélago de Las Guaitecas. De todas las formas de vida ya mencionadas, sólo en las hemicriptófitas se presenta un número considerable de especies adventicias, mientras que en el resto son las especies nativas las que dominan casi absolutamente. Aunque no corresponden a este macroclima, también tienen una representación importante los caméfitos (subarbustos y plantas en cojín) que encuentran hábitat en dunas, roqueríos y turberas, constituyendo la llamada vegetación azonal (Walter 1997). Lo mismo ocurre con los criptófitos, plantas acuáticas en su gran mayoría. Los terófitos (plantas anuales) presentaron 24 especies, mayoritariamente especies adventicias (Tabla 4). Muchas de estas malezas son indicadoras de sequía y por ello colonizan dunas, con sequía edáfica.

Considerando la distribución de las formas de vida en las principales formaciones vegetales (Fig. 4) las especies fanerófitas, trepadoras, epífitas y parásitas alcanzan su mayor frecuencia en los bosques y los matorrales. Por su parte las especies caméfitas son más frecuentes en las turberas y en la vegetación herbácea. Las especies hemicriptófitas alcanzan casi la mitad de la suma de frecuencias en la vegetación herbácea y en los matorrales. En estas dos formaciones es donde las terófitas logran sus más altas frecuencias. Por su parte las criptófitas se distribuyen principalmente en la vegetación herbácea $y$ en las turberas.

Los espectros biológicos para cada isla se muestran en la figura 5. En ellos se pueden observar leves variaciones entre islas, siendo algunas de las más notorias la de los terófitos, con mayores frecuencias en las islas Guamblin e Ipún, y la de los caméfitos, mayoritariamente en las islas Stokes y Kent. La abundancia de terófitos en las islas Guamblin e Ipún puede relacionarse con el grado de alteración antrópica en ellas debido a la frecuencia de visitas y de asentamientos temporales en sus costas. Por otro lado la mayor abundancia de turberas en las islas Stokes y Kent, lo cual puede deberse a su relieve más abrupto y a la diferencia en el estrato 
rocoso subyacente, determina la mayor frecuencia de caméfitos en ellas.

Formaciones vegetales y la biodiversidad ecológica

El paisaje de las islas visitadas está formado por bosques, matorrales, turberas, dunas, pantanos, marismas y vegetación acuática y palustre, todas estas formaciones vegetales ofrecen gran cantidad de hábitats tanto para la fauna como también para la flora (Tabla 6). Uno de los principales elementos de paisaje en las islas estudiadas lo constituye el bosque, con cuatro comunidades vegetales: bosques de tepa-tineo, de mirtáceas, de coihue de Chiloé y de tepú, todos perennifolios, pertenecientes al bosque higrófilo templado o bosque valdiviano en sus subdivisiones de bosque chilote y bosque magallánico perennifolio (Ramírez \& Figueroa 1985). El primero, el bosque de coihue de Chiloé, es el más abundante y está presente en todas las islas visitadas. Al parecer el bosque de mirtáceas podría ser sólo una degradación de este bosques o una etapa en su recuperación, ya que está formado por especies presentes en la comunidad boscosa original (Amigo et al. 2004). El bosque de tepa-tineo se encuentra en mayores altitudes y es más escaso. Bosquetes de ciprés de las Guaitecas aparecen sólo en lugares turbosos de la isla Kent. Si bien las comunidades arbóreas dominadas por tepú son clasificadas generalmente como matorrales debido a su estatura, en las zonas deprimidas de anegamiento edáfico adquiere un porte arbóreo, como es en el caso de la isla Ipún (Ramírez et al. 2006a). Esta comunidad se hace más pequeña pero más abundante en las orillas rocosas de los canales interiores, como lo describen Ramírez \& Álvarez (2008) ${ }^{1}$ para el canal Walker, ubicado en la boca Wickham en el extremo sur del archipiélago.

Dentro de la formación vegetal de matorral se encontraron también 4 comunidades: matorral de ñipa, de chilco, de zarzaparrilla y de calafate. El matorral de chilco está presente sólo en las islas Guamblin e Ipún. Puesto que estos matorrales han sido descritos como estados secundarios de la dinámica de degradación-regeneración del bosque (Amigo et al. 2007), posiblemente indican efectos de tala y quema del bosque, sin embargo no se puede descartar efectos naturales como la fuerte dinámica tectónica en estas latitudes (Fuenzalida 1978, Veblen \& Ashton 1978).

Como integrante importante de este matorral se ha descrito a Chusquea quila, cuya presencia y abundancia había sido mencionada en informes de guardaparques de la CONAF, así como en los relatos de la excursión de Roqueferre (1987, p. 37: "Alcanzamos la lobera norte, después de abrirnos camino con machetes entre nalcas y quilantales"). Su desaparición constituye un misterio y un desafío interesante, ya que ella es una especie altamente resistente a condiciones climáticas y edáficas extremas. En este matoral se encontró también a Gunnera tinctoria coincidente con lo señalado por Hildebrand-Vogel (1988). El matorral de ñipa también sólo se encuentra en Guamblin e Ipún y corresponde a una comunidad nativa que coloniza lugares rocosos del litoral, los que al parecer fueron favorecidos por un levantamiento de las islas durante los sismos de mayo de 1960 (Fuenzalida 1978). En esta comunidad abundan Escallonia rubra y Libertia chilensis. Los matorrales de zarzaparrilla y de calafate sólo se encuentran en la isla Ipún y ambos se presentan como delgadas franjas en el límite del bosque de coihue de Chiloé con pantanos, en el caso del matorral de zarzaparrilla, o con dunas, en el caso del matorral de calafate.

Las turberas abundan en las islas interiores, estando completamente ausentes en Guamblin, donde el bosque dominante no deja espacio a ellas. Las turberas esfagnosas, características por su color rojizo (mallín colorado) están dominadas por Sphagnum magellanicum. Estas turberas son topogénicas, presentándose en bajas altitudes y en depresiones con anegamiento edáfico, incluso se las encuentra colonizando las orillas de lagos oligotróficos, como en el caso de la laguna Enrique, al sur de la isla Ipún (Ramírez et al. 2006a). A mayores altitudes y en lugares rocosos se forman turberas pulvinadas, donde abundan Donatia fascicularis y Lepidothamnus fonckii. Estas turberas más secas, son ombrogénicas ya que dependen del agua que retienen de las precipitaciones (San Martín et al. 2004). Tanto el sustrato dominado por roca sedimentaria como el relieve bastante homogéneo y de

${ }^{1}$ Ramírez, C. \& I. Álvarez 2008. Flora y vegetación terrestre del estero Walker (Región de Aysén, Chile). Informe para Doppler S.A., Valdivia. 27 pp. 
baja altura de las islas Guamblin e Ipún determinan la ausencia de turberas pulvinadas en ellas.

El resto de las formaciones son unidades del paisaje litoral de las islas. Dunas y pantanos están presentes en todas las islas y ellas son colonizadas por neófitas. En la flora nativa de dunas son importantes Calystegia soldanella y Nolana paradoxa, especies de regiones septentrionales más cálidas cuya área conocida se amplía ahora hacia el sur (San Martín et al. 1992). Las marismas son de pequeña extensión se presentan en lugares bajos donde los arroyos desembocan al mar provocando condiciones de anegamiento y también de mayor salinidad, entregando hábitat a halófitos como Cotula coronopifolia y Atriplex chilense (Ramírez et al. 1989). Sin embargo, cabe señalar que pese al contacto directo que tienen estas comunidades con el agua marina, las abundantes precipitaciones reducen considerablemente la salinidad de estos ambientes posibilitando la ocurrencia de especies no halófitas (Roig et al. 1985, Eskuche 2005). La vegetación acuática es escasa y se presenta en lagunas y charcos, siendo importante la presencia de Lemna minuscula un pequeño hidrofito flotante libre que cubría una charca de la isla Guamblin (Ramírez \& San Martín 2006).

Las dunas fueron las formaciones más ricas en especies, alcanzando a 69. A ellas les siguen el bosque de coihue de Chiloé (63 especies), el bosque de mirtáceas (59 especies) y el matorral de ñipa (55 especies). Por su parte las formaciones más pobres en especies fueron el matorral de calafate y el matorral de zarzaparrilla con 7 y 11 especies, respectivamente (Tabla 5).

Las especies adventicias fueron más abundantes en dunas (24 especies), marismas (9 especies), y pantanos ( 9 especies). Sólo 5 especies introducidas se presentan en los matorrales de ñipa y de calafate, 3 especies en el matorral de chilco y en las lagunas, y sólo una especie en el bosque de mirtáceas, en el matorral de zarzaparrilla y en la turbera esfagnosa. Los bosques de tepa-tineo, de coihue de Chiloé y de tepú, así como también la turbera pulvinada no presentan especies adventicias en su flora, lo cual indica el carácter prístino de estas comunidades vegetales en las islas estudiadas (Tabla 5). Por otro lado, el hecho de que las comunidades distribuídas en las costas de las islas contengan una mayor cantidad de neófitos tiene que ver con su accesibilidad y por las condiciones edáficas más favorables para el crecimiento de estas especies.

\section{Afinidad florística}

Sólo 18 especies, alrededor de un $9 \%$ de la flora total, están presentes en las 4 islas estudiadas, entre ellas 5 árboles (Caldcluvia paniculata, Drimys winteri, Lomatia ferruginea, Luma apiculata y Nothofagus nitida), 4 trepadoras (Campsidium valdivianum, Luzuriaga polyphylla, Mitraria coccinea y Pseudopanax laetevirens) y 5 helechos (Blechnum chilense, Blechnum magellanicum, Hymenoglossum cruentum, Hymenophyllum pectinatum y Lophosoria quadripinnata), todas propias de bosques, que es la formación más abundante (Tabla 6). Entre los helechos existen algunos de gran tamaño y también pequeños helechos película que creciendo epífitos sobre los árboles o indican pristinidad. Alrededor de un $23 \%$ de la flora estudiada (48 especies) se restringe a 3 islas, mientras que cerca de un $68 \%$ de ella se restringe a una o dos islas (Fig. 6). Esta distribución de frecuencias indica una baja afinidad florística entre las islas, seguramente debido a la escasa similitud de hábitats ofrecido por el distinto relieve y altitud de ellas, especialmente en las formaciones no boscosas. Una baja similitud entre las comunidades vegetales se puede deducir de la distribución de frecuencias presentadas también en la figura 6 , no existiendo ninguna especie que crezca en más de 8 de las 14 comunidades muestreadas.

Sólo el bosque de coihue de Chiloé, las dunas y los pantanos están presentes en todas las islas estudiadas. Marismas, vegetación acuática, bosque de mirtáceas y turbera esfagnosa faltan en una isla. El resto de las comunidades está presente en 2 o 1 de las islas. Esta diferencia vegetacional entre las islas explica la baja similitud florística entre ellas, ya que la vegetación es la que entrega hábitats para flora y fauna (Tabla 6).

La mayor similitud florística se presentó entre las islas Guamblin e Ipún (Fig. 7). También un semejante nivel de similitud (sobre un $40 \%$ según el indice de Jaccard) se presenta entre las islas Stokes y Kent, mientras todas las otras comparaciones arrojan similitudes bajo un $30 \%$. Estos valores no parecen estar relacionados con la distancia entre las islas, sino más bien con la pertenencia a un mismo sustrato rocoso. Ello se hace mucho más notorio al observar la baja similitud entre las islas Ipún y 
Stokes, pese a ser las más próximas entre sí. Esta disimilitud también se refleja en la ausencia de los matorrales en las islas Stokes e Ipún, sugiriendo una fuerte dependencia entre la geomorfología de las islas, su composición vegetacional y, por ende, su composición florística.

Considerando la composición de las comunidades aquí estudiadas, se observa una buena correspondencia entre la ordenación de ellas según el nMDS y las respectivas formaciones vegetales (Fig. 8). La comunidades boscosas presentan una alta similitud entre ellas, concentrándose en el cuarto inferior izquierdo del diagrama. Los matorrales, por su parte, se dispersan en la parte inferior del diagrama, entre los bosques y el grupo formado por dunas y marismas. Tanto las turberas esfagnosas como las pulvinadas se ubican en el cuarto superior izquierdo del diagrama, claramente separadas del resto de las comunidades, lo cual se relaciona con el alto grado de especialización de las especies que crecen en este tipo de ambientes. El resto de las comunidades no boscosas (marismas, dunas, pantanos y lagunas) se concentran en el lado derecho del diagrama y muestran una alta heterogeneidad. De acuerdo al análisis realizado el primer eje horizontal más importante, podría asimilarse a un gradiente de temperatura que aumentaría de izquierda a derecha, y el segundo vertical, correspondería a un gradiente de humedad (o anegamiento) que aumenta de abajo hacia arriba.

\section{Clasificación de la vegetación}

La vegetación potencial de las islas estudiadas corresponde al llamado bosque higrófilo templado norpatagónico (Fuenzalida 1965, Pisano 1981) definido posteriormente por Quintanilla (1981) como pluviselva norpatagónica. Más tarde Quintanilla (1989) entrega un esquema de la zonación de la vegetación en la isla Guamblin y plantea la presencia de bosques y matorrales siempreverdes de Aisén insular, los que sitúa en un mapa. Siguiendo a Quintanilla (1981), Gajardo (1994) la nombra zona de matorrales siempreverdes oceánicos, mencionando tentativamente algunas asociaciones vegetales sin entregar tablas de vegetación. Este esquema vegetacional fue mejorado por Luebert \& Pliscoff (2006). Concordando con estos autores la vegetación estudiada corresponde efectivamente a un bosque siempreverde, florísticamente no muy diferente del llamado bosque valdiviano en su subdivisión de Bosque chilote propuesta por Ramírez \& Figueroa (1985).

Una clasificación más detallada podría hacerse desde el punto de vista fitosociológico (e.g. Oberdorfer 1960, Roig 1998). Pese a que en este trabajo no se registró la abundancia de las especies en cada comunidad, información valiosa al momento de clasificar comunidades en asociaciones vegetales, es posible suponer la correspondencia de estas comunidades a asociaciones o sintaxa descritas en la literatura (Tabla 7). La mayoría de las comunidades boscosas y arbustivas pueden ser clasificadas dentro de la clase Wintero-Nothofagetea (Oberdorfer 1960). Sin embargo, las comunidades arbustivas son más difíciles de asignar a alguna asociación definida debido a sus composiciones un poco atípicas, faltando algunos elementos importantes de ellas como es el caso de las especies Aristotelia chilensis y Chusquea quila. Al respecto Amigo et al. (2007) ofrecen una revisión de estas comunidades en Chile. Otro tanto ocurre con las asociaciones herbáceas (dunas, pantanos, marismas y lagunas), en las cuales se incluyen varias asociaciones, incluso algunas aún no descritas, lo cual se corresponde con la alta variabilidad observada en el diagrama de ordenación. Tanto por la ubicación geográfica y el ambiente que ocupan estas comunidades así como por su composición florística es posible que algunas de ellas se puedan agrupar dentro de la clase Deschampsio-Asteretea, propuesta por Roig et al. (1985) y posteriormente renombrada como Leptinelletalia scariosae por Eskuche (2005).

\section{Dinámica vegetacional}

Como lo plantean Szeicz et al. (2003) la vegetación actual del archipiélago de Los Chonos es el resultado de una serie de eventos ocurridos en el tiempo geológico y reciente, influenciado además, por la acción antrópica. La vegetación potencial zonal concordante con el macroclima (Walter 1997) original de las islas estudiadas estaría formada por los bosques de coihue de Chiloé, de tepa-tineo, de mirtáceas y de tepú, que corresponderían a la formación bosque higrófilo templado norpatagónico. La primera comunidad, bosque de coihue de Chiloé es la dominante y al ser destruida es reemplazada por los matorrales de chilco y de zarzaparrilla, que junto con el matorral de calafate, serían comunidades secundarias de reemplazo. Como se dijo, también 
el bosque de mirtáceas podría ser una degradación de él. No obstante los efectos antrópicos observados durante la expedición, también debe ser tomada en cuenta la dinámica generada por causas naturales, de magnitudes catastróficas como lo fue el sismo de 1960 (Fuenzalida 1978, Roqueferre 1987). Dichos efectos son patentes en las costas norte y este de la isla Guamblin, donde grandes extensiones de matorrales crecen sobre un sustrato emergido desde el fondo marino, razón por la cual es frecuente encontrar una costra calcárea y restos de moluscos bajo el delgado suelo orgánico. También las profundas grietas que surcan estas plataformas rocosas, en las cuales comúnmente se forman las lagunas dominadas por Lemna minima, son posiblemente otro resultado de ello. Por ende, estas formaciones arbustivas constituyen estados de sucesión primaria, de origen natural. Precisamente esta dinámica sucesional podría explicar la ausencia de Chusquea quila, especie que a juzgar por los informes de CONAF y por las menciones de Roqueferre (1987) era bastante frecuente en esta isla, aunque se puede olvidar que la quila es un componente del sotobosque.
Estado de conservación y perspectivas de estudio

El estado de conservación de la vegetación de las islas estudiadas es aceptable, ya que la ocasional actividad humana se concentra en el litoral de ellas y no en las comunidades boscosas y turbosas del interior. Aunque en su flora hay algunos problemas de conservación, especialmente en helechos, esto no representa una dificultad apremiante, ya que ella también está representada al norte y al sur de la región de Aisén. Sin embargo, existen algunos problemas que deben ser monitoreados, como son la inexplicable desaparición de la quila, cuya presencia había sido informada por guardaparques en informes no muy antiguos.

Debido a que en estas islas es posible encontrar tanto comunidades boscosas prístinas, como estados sucesionales primarios, además de que los efectos de alteración antrópica se presentan en forma localizada, es necesario hacer estudios más intensivos para determinar el grado de peligro que ofrecen las actividades humanas sobre el desarrollo de las dinámicas sucesionales, especialmente en la isla Guamblin.

TABLA 1. Distribución en clases de la flora muestreada en las islas por clase.

\begin{tabular}{lrc}
\hline \multicolumn{1}{c}{ Clase } & Especies & Porcentaje \\
\hline Bryopsida (Musgos) & 1 & 0,49 \\
Lycopodiopsida (Licopodios) & 1 & 0,49 \\
Polypodiopsida (Helechos) & 29 & 14,15 \\
Pinopsida (Coníferas) & 3 & 1,46 \\
Magnoliopsida (Dicotiledóneas) & 121 & 59,02 \\
Liliopsida (Monocotiledóneas) & 50 & 24,39 \\
\hline Total & 205 & 100,00 \\
\hline
\end{tabular}

TABLA 2. Superficie, número de especies y días de muestreo en cada una de las islas estudiadas.

\begin{tabular}{lcccc}
\hline \multicolumn{1}{c}{ Islas: } & Guamblin & Ipún & Stokes & Kent \\
\hline Superficie (ha) & 15.912 & 8.418 & 11.135 & 9.000 \\
Especies adventicias & 20 & 23 & 4 & 5 \\
Especies nativas & 106 & 110 & 85 & 72 \\
Total especies & 126 & 133 & 89 & 77 \\
\hline Días de muestreo & 7 & 3 & 2 & 1 \\
\hline
\end{tabular}

TABLA 3. Modelos de regresión lineal del número de especies encontradas en las islas en función a su superficie, al esfuerzo de muestreo y a la cantidad de comunidades encontradas en ellas.

\begin{tabular}{lccc}
\hline \multicolumn{1}{c}{ Variables } & Intercepto & Pendiente & $\mathrm{R}^{2}$ \\
\hline Superficie (ha) & 79,349 & 0,0024 & 0,0901 \\
Días de muestreo & 82,012 & 7,4578 & 0,5109 \\
\hline Número de comunidades & 40,267 & 7,1333 & 0,4224 \\
\hline
\end{tabular}


TABLA 4. Formas de vida y origen de las especies presentes en las islas.

\begin{tabular}{lrccccccc}
\hline Origen & $\mathrm{Ph}$ & $\mathrm{Cl}$ & $\mathrm{Ep}$ & $\mathrm{Ch}$ & $\mathrm{H}$ & $\mathrm{Cr}$ & $\mathrm{T}$ & Total \\
\hline Adventicias & 1 & - & - & 1 & 12 & 4 & 14 & 32 \\
Nativas & 36 & 10 & 19 & 21 & 66 & 10 & 10 & 172 \\
\hline Total & 37 & 10 & 19 & 22 & 78 & 14 & 24 & 204 \\
\hline
\end{tabular}

Formas de vida: $\mathrm{Ph}$ - fanerófitas, $\mathrm{Cl}$ - trepadoras, Ep - epífitas y parásitas, $\mathrm{Ch}$ - caméfitas, $\mathrm{H}$ hemicriptófitas, $\mathrm{Cr}$ - criptófitas, $\mathrm{T}$ - terófitas.

TABLA 5. Riqueza florística de las comunidades estudiadas y origen de las especies.

\begin{tabular}{lccc}
\hline Comunidades & \multicolumn{3}{c}{ Especies } \\
\cline { 2 - 3 } & nativas & introducidas & total \\
\hline Bosque de tepa-tineo & 34 & - & 34 \\
Bosque de mirtáceas & 58 & 1 & 59 \\
Bosque de coihue de Chiloé & 63 & - & 63 \\
Bosque de tepú & 22 & - & 22 \\
Matorral de ñipa & 50 & 5 & 55 \\
Matorral de chilco & 29 & 3 & 32 \\
Matorral de zarzaparrilla & 10 & 1 & 11 \\
Matorral de calafate & 2 & 5 & 7 \\
Dunas & 45 & 24 & 69 \\
Marismas & 27 & 9 & 36 \\
Pantanos & 36 & 9 & 45 \\
Turbera esfagnosa & 30 & 1 & 31 \\
Turbera pulvinada & 20 & - & 20 \\
Lagunas & 16 & 3 & 19 \\
\hline Total & 173 & 32 & 205 \\
\hline
\end{tabular}

TABLA 6. Distribución de las comunidades en las islas estudiadas.

\begin{tabular}{|c|c|c|c|c|c|}
\hline \multirow[t]{2}{*}{ Comunidades } & \multicolumn{4}{|c|}{ Islas } & \multirow[t]{2}{*}{ Frecuencia } \\
\hline & Guamblin & Ipún & Kent & Stokes & \\
\hline Bosque de tepa-tineo & & + & + & & 2 \\
\hline Bosque de mirtáceas & + & + & + & & 3 \\
\hline Bosque de coihue de Chiloé & + & + & + & + & 4 \\
\hline Bosque de tepú & & + & + & + & 3 \\
\hline Matorral de ñipa & + & + & & & 2 \\
\hline Matorral de chilco & + & + & & & 2 \\
\hline Matorral de zarzaparrilla & & + & & & 1 \\
\hline Matorral de calafate & & + & & & 1 \\
\hline Dunas & + & + & + & + & 4 \\
\hline Marismas & + & + & & + & 3 \\
\hline Pantanos & + & + & + & + & 4 \\
\hline Turbera esfagnosa & & + & + & + & 3 \\
\hline Turbera pulvinada & & & + & + & 2 \\
\hline Lagunas & + & + & & + & 3 \\
\hline Total (15 comunidades) & 8 & 13 & 8 & 8 & \\
\hline
\end{tabular}


TABLA 7. Asociaciones correspondientes a las unidades vegetales estudiadas.

\begin{tabular}{|c|c|c|}
\hline Formación & Comunidad & Sintaxa \\
\hline \multirow[t]{4}{*}{ Bosque } & de tepa-tineo & $\begin{array}{l}\text { Laurelio philippianae-Weinmannietum } \\
\text { trichospermae Oberdorfer } 1960\end{array}$ \\
\hline & de mirtáceas & - \\
\hline & de coihue de Chiloé & Luzuriago polyphyllae-Nothofagetum nitidae Amigo et al. 2004 \\
\hline & de tepú & Tepualietum stipulariae Hueck 1978 \\
\hline \multirow[t]{4}{*}{ Matorral } & de ñipa & Griselinio jodinifoliae-Pernettyetum poeppigii ${ }^{(1)}$ Hildebrand 1983 \\
\hline & de chilco & $\begin{array}{l}\text { Fuchsio magellanicae-Chusqueetum quilae } e^{(2)} \text { Hildebrand } 1983 \\
\text { Aristotelio chilensis-Fuchsietum magellanicae }{ }^{(3)} \text { Oberdorfer } 1960\end{array}$ \\
\hline & de zarzaparrilla & - \\
\hline & de calafate & Berberidion buxifoliae Oberdorfer 1960 \\
\hline \multirow[t]{2}{*}{ Turbera } & esfagnosa & Myrteolo damaeonchii-Sphagnetalia magellanici Oberdorfer 1960 \\
\hline & pulvinada & Drosero uniflori-Donatietum fascicularis Ramírez 1968 \\
\hline Dunas & no definida & - \\
\hline Pantanos & varias & - \\
\hline Marismas & no definida & - \\
\hline Lagunas & varias & - \\
\hline
\end{tabular}

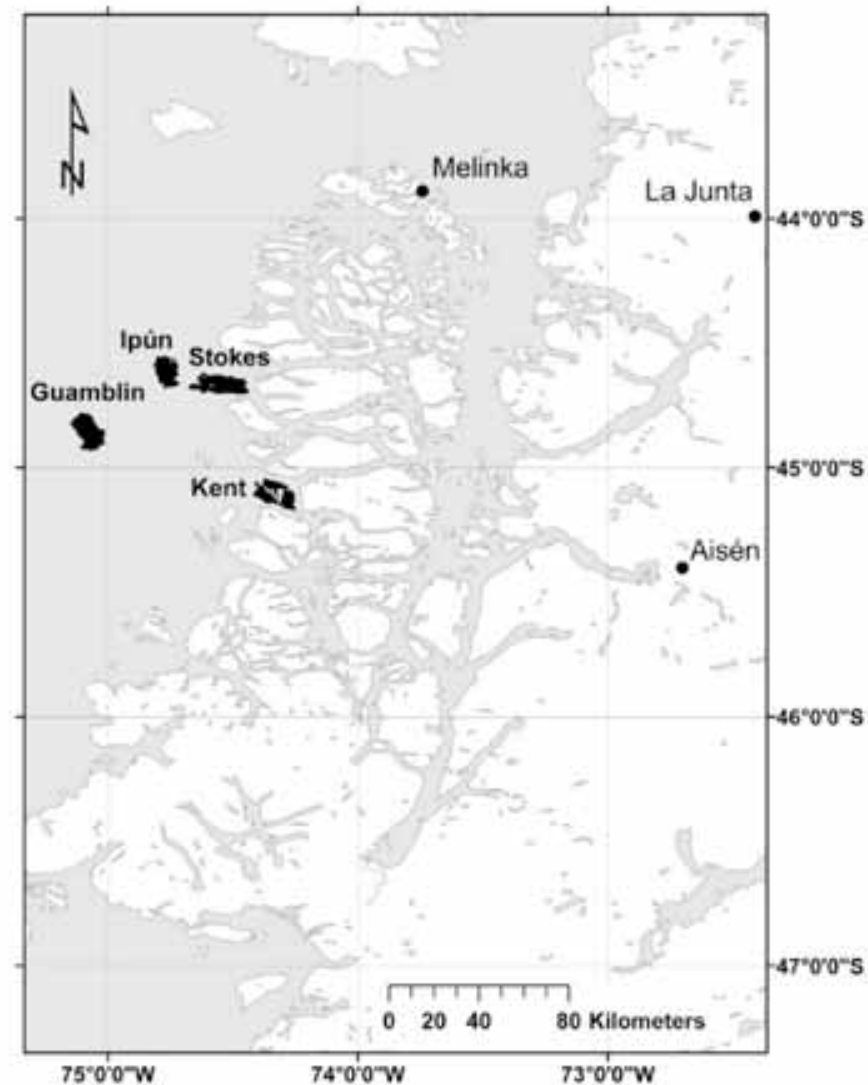

Fig. 1. Ubicación de las islas estudiadas en el archipiélago de Los Chonos. 


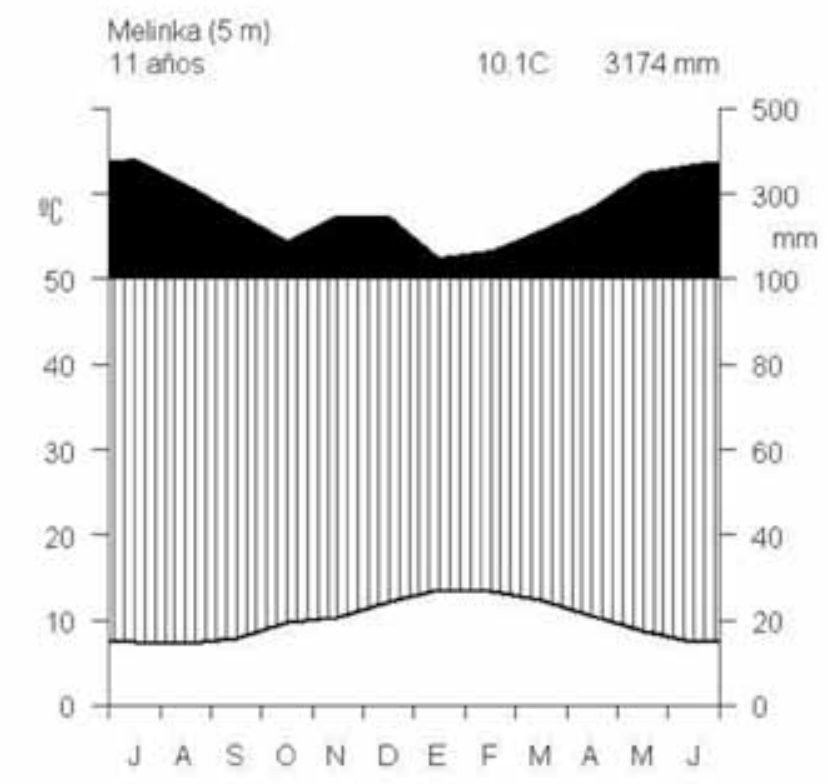

Fig. 2. Diagrama climático de Melinka. Fuente: Hajek \& di Castri (1975).

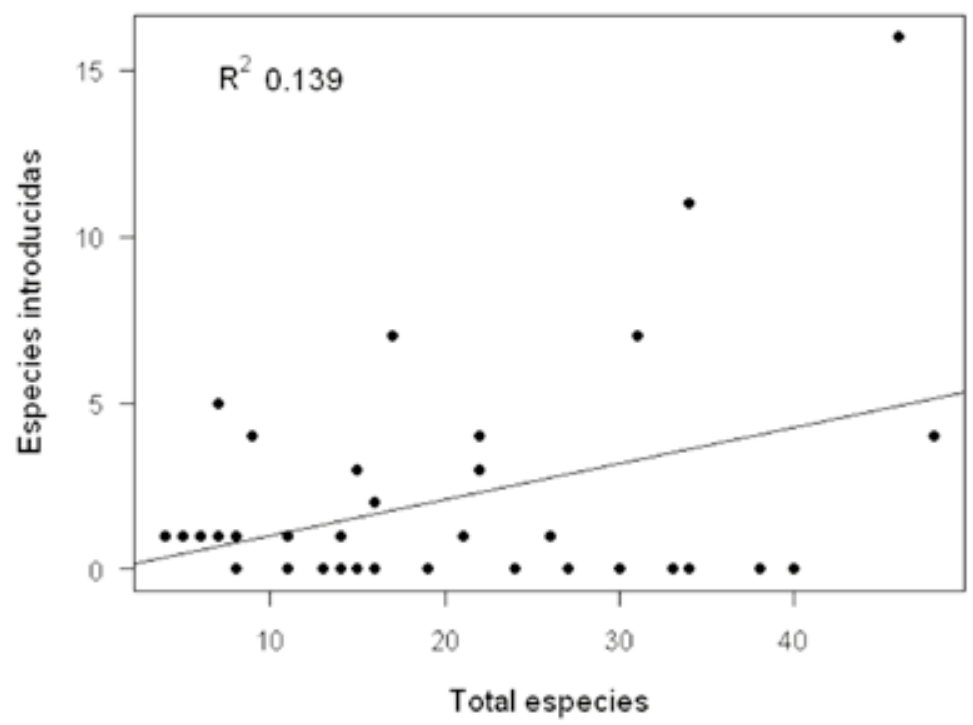

Fig. 3. Número de especies introducidas versus número total de especies muestradas en cada comunidad de cada isla. 

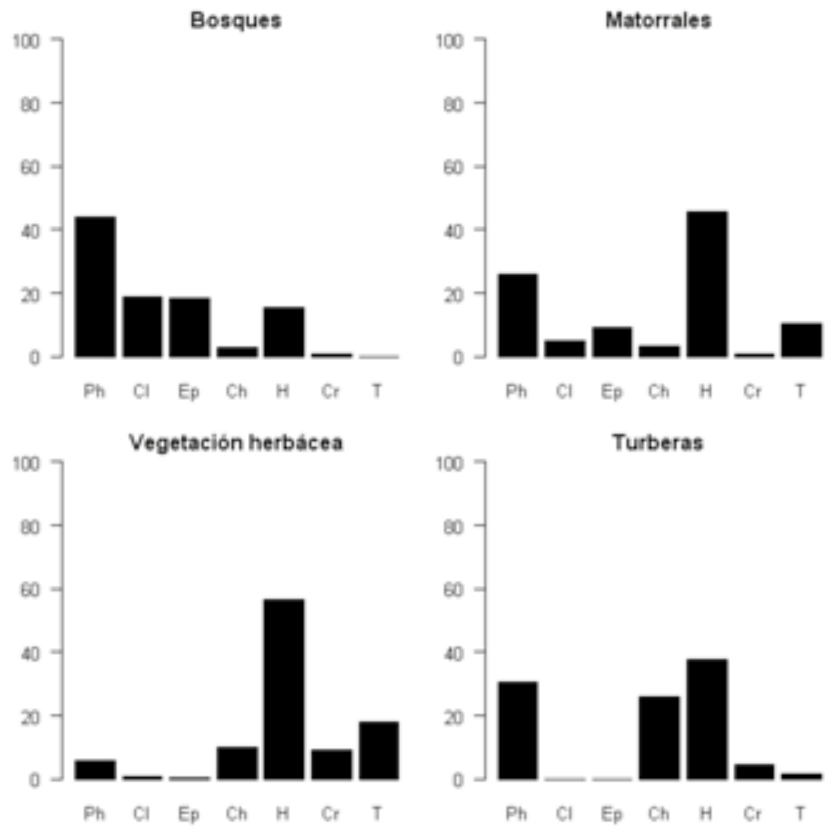

Fig. 4. Espectros biológicos de los distintos tipos de vegetación estudiados en este trabajo. Frecuencias relativas de las formas de vida: $\mathrm{Ph}$ - fanerófitas, $\mathrm{Cl}$ - trepadoras, Ep - epífitas y parásitas, $\mathrm{Ch}$ - caméfitas, $\mathrm{H}$ - hemicriptófitas, $\mathrm{Cr}$ - criptófitas, T - terófitas.
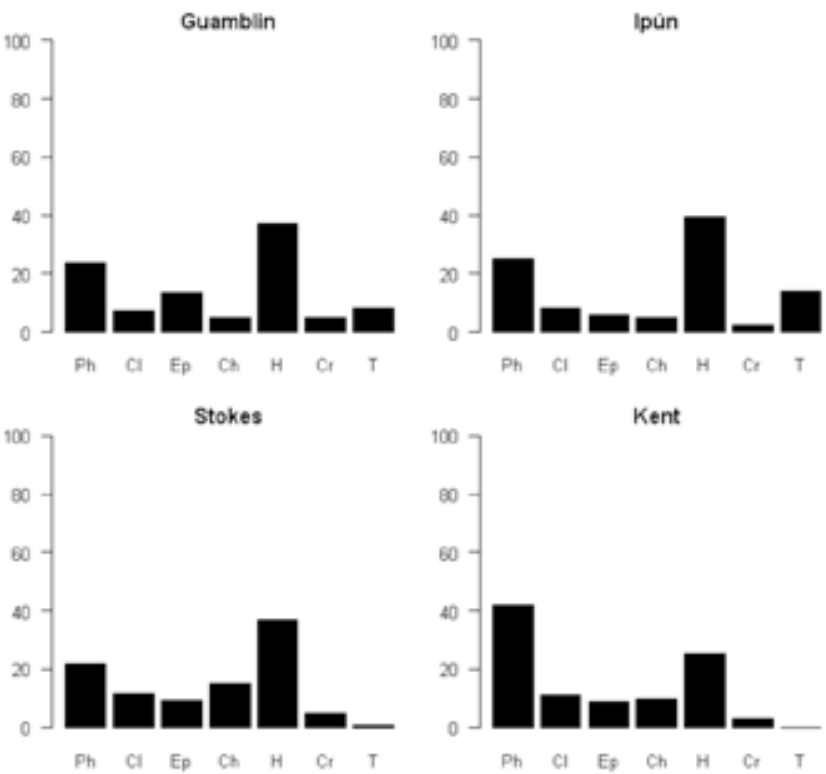

Fig. 5. Espectros biológicos de las islas estudiadas en este trabajo.

Frecuencias relativas de las formas de vida: $\mathrm{Ph}$ - fanerófitas, $\mathrm{Cl}$ - trepadoras, $\mathrm{Ep}$ - epífitas y parásitas, $\mathrm{Ch}$ - caméfitas, $\mathrm{H}$ - hemicriptófitas, $\mathrm{Cr}$ - criptófitas, T - terófitas. 

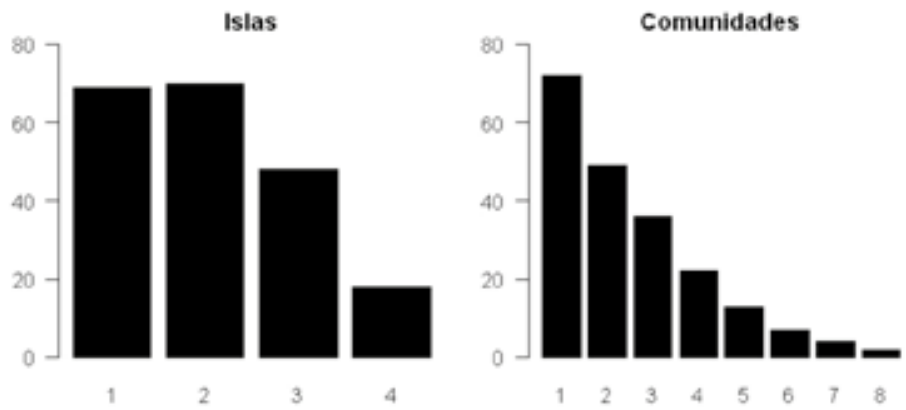

Fig. 6. Distribución de la frecuencia de las especies en las islas (izquierda) y en las comunidades (derecha).

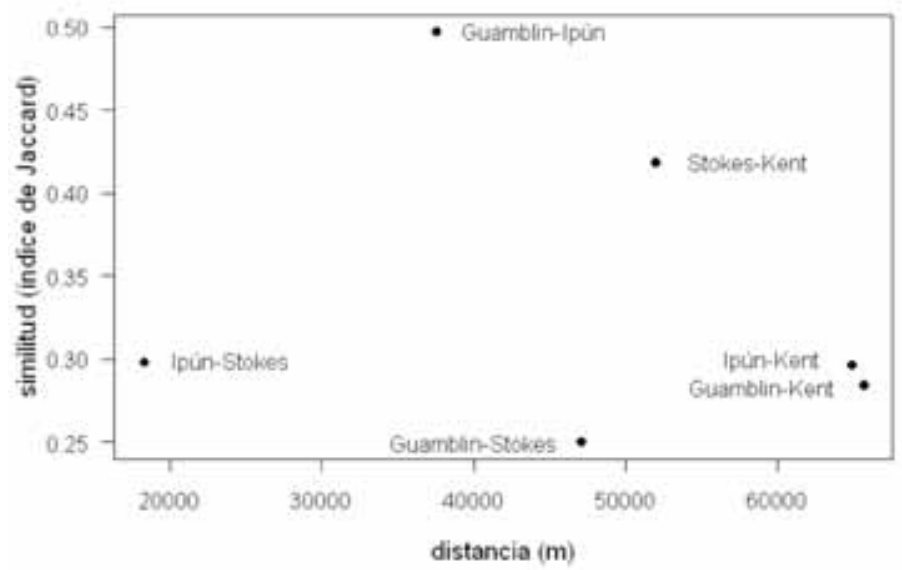

Fig. 7. Similitud floristica versus distancia geográfica entre las islas estudiadas.

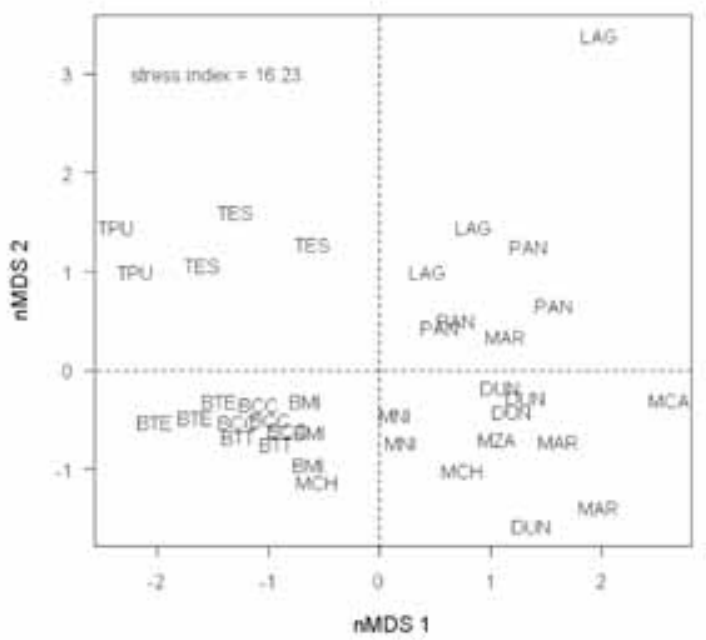

Fig. 8. Diagrama de ordenación según nMDS (non-metric multidimensional scaling) usando el índice de similitud de Jaccard y dos dimensiones.

Símbolos: BTT - Bosque de tepa-tineo, BMI - Bosque de mirtáceas, BCC - Bosque de coihue de Chiloé, BTE - Bosque de tepú, MNI - Matorral de ñipa, MCH - Matorral de chilco, MZA - Matorral de zarzaparrilla, MCA Matorral de calafate, DUN - Dunas, MAR - Marismas, PAN - Pantanos, TES - Turbera esfagnosa, TPU - Turbera pulvinada, LAG - Lagunas. 
Anexo 1. Caracterización de la flora vascular de las islas estudiadas (Abreviaturas se muestran al final de la tabla).

\begin{tabular}{|c|c|c|c|c|}
\hline Clases y especies & Familia & Nombre común & $\mathrm{O}$ & FV \\
\hline Bryopsida (Musgos) & & & & \\
\hline $\begin{array}{l}\text { Sphagnum magellanicum Bridg. } \\
\text { Lycopodiopsida (Licopodios) }\end{array}$ & Sphagnaceae & Pon-pón & $\mathrm{N}$ & - \\
\hline Lycopodium gayanum Remy & Lycopodiaceae & Licopodio & $\mathrm{N}$ & $\mathrm{Cr}$ \\
\hline $\begin{array}{l}\text { Polypodiopsida (Helechos) } \\
\text { Adiantum chilense Kaulf }\end{array}$ & Adiantaceae & Culantrillo & $\mathrm{N}$ & $\mathrm{H}$ \\
\hline Asplenium dareoides A.N.Desv. & Aspleniaceae & Filu-lahuén & $\mathrm{N}$ & Ep \\
\hline Asplenium obtusatum G.Forster & & - & $\mathrm{N}$ & Ep \\
\hline Asplenium trilobum Cav. & & - & $\mathrm{N}$ & Ep \\
\hline Blechnum chilense (Kaulf.) Mett. & Blechnaceae & Quil-quíl & $\mathrm{N}$ & $\mathrm{H}$ \\
\hline Blechnum magellanicum (A.N.Desv.) Mett. & & Palmerita & $\mathrm{N}$ & $\mathrm{Ph}$ \\
\hline Blechnum microphyllum (Goldm.) Morton & & - & $\mathrm{N}$ & $\mathrm{H}$ \\
\hline Blechnum mochaenum Kunkel & & Palmilla & $\mathrm{N}$ & $\mathrm{H}$ \\
\hline Ctenitis spectabilis (Kaulf.) Kunkel & Aspidiaceae & & $\mathrm{N}$ & $\mathrm{H}$ \\
\hline Gleichenia quadripartita (Pir.) Moore & Gleicheniaceae & Hierba loza & $\mathrm{N}$ & $\mathrm{H}$ \\
\hline Gleichenia squamulosa (A.N.Desv.)Moore & & & $\mathrm{N}$ & $\mathrm{H}$ \\
\hline Grammitis magellanica A.N.Desv. & Grammitidaceae & - & $\mathrm{N}$ & Ep \\
\hline Hymenoglossum cruentum (Cav.) K.Presl. & Hymenophyllaceae & Sanguinaria & $\mathrm{N}$ & Ep \\
\hline Hymenophyllum caudiculatum Mart. & Hymenophyllaceae & Helecho película & $\mathrm{N}$ & Ep \\
\hline Hymenophyllum darwinii (Hook.f.) van den Bosch & & & $\mathrm{N}$ & Ep \\
\hline Hymenophyllum dentatum Cav. & & & $\mathrm{N}$ & Ep \\
\hline Hymenophyllum dicranotrichum (K.Presl.) Sadeb. & & & $\mathrm{N}$ & Ep \\
\hline Hymenophyllum krauseanum Phil. & & & $\mathrm{N}$ & Ep \\
\hline Hymenophyllum pectinatum Cav. & & & $\mathrm{N}$ & Ep \\
\hline Hymenophyllum plicatum Kaulf. & & & $\mathrm{N}$ & Ep \\
\hline Hymenophyllum secundum Hook. et Grev. & & & $\mathrm{N}$ & Ep \\
\hline Hymenophyllum seselifolium K.Presl. & & & $\mathrm{N}$ & Ep \\
\hline Hymenophyllum tortuosum Hook. et Grev. & & & $\mathrm{N}$ & Ep \\
\hline Lophosoria quadripinnata (J.F.Gmel.) C.Chr. & Lophosoriaceae & Ampe & $\mathrm{N}$ & $\mathrm{H}$ \\
\hline Polypodium feuillei Bert. & Polypodiaceae & Calahuala & $\mathrm{N}$ & Ep \\
\hline Polystichum plicatum (Poepp. ex Kunze) Hicken & Aspidiaceae & - & $\mathrm{N}$ & $\mathrm{H}$ \\
\hline Rumorha adiantiformis (G.Forster) Ching. & & Helecho & $\mathrm{N}$ & $\mathrm{H}$ \\
\hline Schizaea fistulosa Labill. & Schizaeaceae & Helecho alambre & $\mathrm{N}$ & $\mathrm{H}$ \\
\hline $\begin{array}{l}\text { Serpyllopsis caespitosa (Gaud.) C.Chris. } \\
\text { Pinopsida (Coníferas) }\end{array}$ & Hymenophyllaceae & Helecho película & $\mathrm{N}$ & $\mathrm{H}$ \\
\hline Lepidothamnus fonckii (Phil.) Ball. & Podocarpaceae & Ciprés enano & $\mathrm{N}$ & $\mathrm{Ch}$ \\
\hline Pilgerodendron uviferum (D.Don) Florin & Cupressaceae & $\begin{array}{l}\text { Ciprés de Las } \\
\text { Guaitecas }\end{array}$ & $\mathrm{N}$ & $\mathrm{Ph}$ \\
\hline $\begin{array}{l}\text { Podocarpus nubigena Lindl. } \\
\text { Magnoliopsida (Dicotiledóneas) }\end{array}$ & Podocarpaceae & Mañío macho & $\mathrm{N}$ & $\mathrm{Ph}$ \\
\hline Acaena ovalifolia Ruiz et Pav. & Rosaceae & Cadillo & $\mathrm{N}$ & $\mathrm{H}$ \\
\hline Ambrosia chamissonis (Less.) Greene & Asteraceae & Dicha grande & $\mathrm{N}$ & $\mathrm{Ch}$ \\
\hline Amomyrtus luma (Mol.) Legr. et Kaus. & Myrtaceae & Luma & $\mathrm{N}$ & $\mathrm{Ph}$ \\
\hline Amomyrtus meli (Phil.) Legr. et Kaus. & & Meli & $\mathrm{N}$ & $\mathrm{Ph}$ \\
\hline Anagallis alternifolia Cav. & Primulaceae & Pimpinela & $\mathrm{N}$ & $\mathrm{Ch}$ \\
\hline Apium australe Thovars. & Apiaceae & Apio silvestre & $\mathrm{N}$ & $\mathrm{H}$ \\
\hline
\end{tabular}


Aster vahlii (Gaud.) Hook. et Arn.

Asteranthera ovata (Cav.) Hanst.

Atriplex chilensis Colla

Azara lanceolata Hook.f.

Baccharis sagittalis (Less.) DC.

Berberis buxifolia Lam.

Berberis ilicifolia L.f.

Brassica rapa L.

Calceolaria sp.

Caldcluvia paniculata (Cav.) D.Don

Callitriche palustris L.

Callitriche terrestris Rafin.

Calystegia sepium (L.) R.Br.

Calystegia soldanella (L.) Roem. et Schult.

Campsidium valdivianum (Phil.) Skottsb.

Cardamine geraniifolia (Poir.) DC.

Cerastium holosteoides Fries

Cirsium vulgare (Savi) Ten.

Colobanthus quitensis

(Humb., Bonpl. et Kunth) Bartl.

Coronopus didymus (L.) J.E.Sm.

Cotula coronopifolia L.

Cotula scariosa (Cass.) Franchet

Desfontainia spinosa D.Don

Donatia fascicularis J.R. Forster et G.Forster

Drimys winteri J.R. Forster et G.Forster

Drosera uniflora Willd.

Dysopsis glechomoides (A.Rich.) Muell-Arg.

Embothrium coccineum J.R. Forster et G.Forster

Empetrum rubrum Vahl. ex Willd.

Epilobium puberulum Hook. et Arn.

Erigeron karwinskianus DC.

Escallonia rubra (Ruiz et Pav.) Pers.

Euphorbia portulacoides L.

Fragaria chiloensis (L.) Dutch.

Fuchsia magellanica Lam.

Galium aparine L.

Gamochaeta americana (Mill.) Wedd.

Gaultheria phillyreifolia (Pers.) Sleumer

Gnaphalium cheiranthifolium Lam.

Griselinia racemosa (Phil.) Taub.
Asteraceae

Gesneriaceae

Chenopodiaceae

Flacourtiaceae

Asteraceae

Berberidaceae

Brassicaceae

Scrophulariaceae

Cunoniaceae

Callitrichaceae

Convolvulaceae

Bignoniaceae

Brassicaceae

Caryophyllaceae

Asteraceae

Caryophyllaceae

Brassicaceae

Asteraceae

Desfontainiaceae

Donatiaceae

Winteraceae

Droseraceae

Euphorbiaceae

Proteaceae

Empetraceae

Onagraceae

Asteraceae

Saxifragaceae

Euphorbiaceae

Rosaceae

Onagraceae

Rubiaceae

Asteraceae

Ericaceae

Asteraceae

Cornaceae
Margarita del

pantano

Estrellita

Cachiyuyo

Corcolén

Verbena de tres

esquinas

Calafate

Michay

Yuyo

Zapatito de la

virgen

Tiaca

Estrella de agua

Huenchecó

Correhuela de

pantano

Oreja de ratón $\quad \mathrm{N} \quad \mathrm{Cl}$

Voqui de canasta $\mathrm{N} \quad \mathrm{Cl}$

Berrito

Cuernecito

Cardo negro

Colobanto

Mastuerzo

Botón de oro

africano

Paladar

de chancho

Taique

Donatia

Canelo

Rocío de sol

-

Notro

Brecillo

Epilobio

Margarita

mexicana

Nipa

Pichoga de arena

Frutilla

Chilco

Lengua de gato

Vira-vira rojo

Chaura macho

Vira-vira amarillo

Yelmo
$\mathrm{N} \quad \mathrm{Ch}$

$\mathrm{N} \quad \mathrm{Cl}$

$\mathrm{N}$ T

$\mathrm{N} \quad \mathrm{Ph}$

$\mathrm{N} \quad \mathrm{Ph}$

$\mathrm{N} \quad \mathrm{Ph}$

$\mathrm{N} \quad \mathrm{Ph}$

A $T$

$\mathrm{N} \quad \mathrm{H}$

$\mathrm{N} \quad \mathrm{Ph}$

A $\mathrm{Cr}$

$\mathrm{N} \quad \mathrm{Cr}$

A $\mathrm{Cr}$

$\mathrm{N} \mathrm{T}$

A $T$

A $T$

$\mathrm{N} \quad \mathrm{Ch}$

A $\mathrm{T}$

A $\mathrm{H}$

$\mathrm{N} \mathrm{H}$

$\mathrm{N} \quad \mathrm{Ph}$

$\mathrm{N} \quad \mathrm{Ch}$

$\mathrm{N} \quad \mathrm{Ph}$

$\mathrm{N} H$

$\mathrm{N} \quad \mathrm{H}$

$\mathrm{N} \quad \mathrm{Ph}$

$\mathrm{N}$ Ch

$\mathrm{N}$ T

A $\mathrm{T}$

$\mathrm{N} \quad \mathrm{Ph}$

$\mathrm{N}$ Ch

$\mathrm{N} H$

$\mathrm{N} P h$

A $T$

$\mathrm{N}$ T

$\mathrm{N} \quad \mathrm{Ph}$

$\mathrm{N} H$

$\mathrm{N} \quad \mathrm{Cl}$ 
Griselinia ruscifolia (Clos) Taub.

Griselinia scandens (Ruiz et Pav.) Taub.

Gunnera magellanica Lam.

Gunnera tinctoria (Mol.) Mirb.

Hebe elliptica (G.Forster) Pennell.

Hydrangea serratifolia (Hook. et Arn.) F.Phil.

Hydrocotyle chamaemorus Cham. et Schlecht.

Hydrocotyle ranunculoides L.f.

Lathyrus japonicus Willd.

Laurelia philippiana Looser

Lilaeopsis macloviana (Gaud.) A.W.Hill.

Limosella australis R.Br.

Lobelia alata (Wedd.) Lammers

Lomatia ferruginea (Cav.) R.Br.

Luma apiculata (DC.) Burret

Lycopus europaeus L.

Lythrum portula L.

Margyricarpus pinnatus (Lam.) O.K.

Maytenus magellanica (Lam.) Hook.f.

Misodendrum macrolepis Phil.

Misodendrum punctulatum Banks ex DC.

Mitraria coccinea Cav.

Myoschilos oblonga Ruiz et Pav.

Myosotis scorpioides L.

Myrceugenia ovata (Hook. et Arn.) Berg.

Myrceugenia planipes (Hook. et Arn.) Berg.

Myriophyllum quitense Humb., Bonpl. et Kunth

Myrteola nummularia (Poir.) Berg.

Nertera granadensis (Mutis et L.f.) Druce

Nolana paradoxa Lindl.

Nothofagus betuloides (Mirb.) Oerst.

Nothofagus nitida (Phil.) Krasser

Ourisia coccinea (Cav.) Pers.

Ourisia ruelloides (L.f.) O.K.

Ovidia pillopillo (Gay) Meisn.

Pernettya insana (Mol.) Gunckel

Pernettya mucronata (L.f.) Gaud. ex Spreng.

Pernettya pumila (L.f.) Hook.

Pilea elliptica Hook.f.

Pinguicula antarctica Vahl.

Plantago australis Lam.

Plantago grandiflora Meyen

Plantago truncata Cham. et Schlecht.

Polygonum sanguinaria Remy

Potentilla anserina L.

Pseudopanax laetevirens (Gay) Franchet

Gunneraceae
Scrophulariaceae
Hydrangeaceae
Apiaceae

Fabaceae

Monimiaceae

Apiaceae

Scrophulariaceae

Campanulaceae

Proteaceae

Myrtaceae

Lamiaceae

Lythraceae

Rosaceae

Celastraceae

Misodendraceae

Gesneriaceae

Santalaceae

Boraginaceae

Myrtaceae

Haloragaceae

Myrtaceae

Rubiaceae

Nolanaceae

Fagaceae

Scrophulariaceae

Scrophulariaceae

Thymelaeaceae

Ericaceae

Urticaceae

Lentibulariaceae

Plantaginaceae

Polygonaceae

Rosaceae

Araliaceae
Dinacho

Nalca

Hebe

Laurela

Tembladerilla

Hierba de la

plata

Clarincillo

Tepa

-

Limosela

Romerillo

Arrayán

Pata de lobo

Romerillo rojo

Perlilla

Leñadura

Injerto

Botellita

Orocoipo

No me olvides

Arrayán chico

Picha-picha

Pinito de agua

Daudapo

Rucachucao

Suspiro de duna

Coihue

de Magallanes

Coihue de Chiloé

Orisia

Pillo-pillo

Hued-hued

Chaura

Chaurilla

Pilea

Violeta del

pantano

Llantén peludo

Llantén marino

Sanguinaria

Canelilla

Traumén
$\mathrm{N} \quad \mathrm{Cl}$

$\mathrm{N} \quad \mathrm{Cl}$

$\mathrm{N} H$

$\mathrm{N} H$

$\mathrm{N} \quad \mathrm{Ph}$

$\mathrm{N} \quad \mathrm{Cl}$

$\mathrm{N} \quad \mathrm{H}$

A $\mathrm{Cr}$

$\mathrm{N}$ T

$\mathrm{N} \quad \mathrm{Ph}$

$\mathrm{N}$ Cr

$\mathrm{N}$ T

$\mathrm{N}$ Ch

$\mathrm{N} \quad \mathrm{Ph}$

$\mathrm{N} \quad \mathrm{Ph}$

A $\mathrm{Cr}$

A $\mathrm{H}$

$\mathrm{N}$ Ch

$\mathrm{N} \quad \mathrm{Ph}$

$\mathrm{N} P a$

$\mathrm{N} \mathrm{Pa}$

$\mathrm{N} \quad \mathrm{Cl}$

$\mathrm{N} P h$

A $\mathrm{H}$

$\mathrm{N} \quad \mathrm{Ph}$

$\mathrm{N} \quad \mathrm{Ph}$

$\mathrm{N}$ Cr

$\mathrm{N}$ Ch

$\mathrm{N} H$

N T

$\mathrm{N} P h$

$\mathrm{N} \quad \mathrm{Ph}$

$\mathrm{N} H$

$\mathrm{N} H$

$\mathrm{N} \quad \mathrm{Ph}$

$\mathrm{N} P h$

$\mathrm{N} P h$

$\mathrm{N} \quad \mathrm{Ph}$

$\mathrm{N} H$

$\mathrm{N} \mathrm{H}$

$\mathrm{N} \quad \mathrm{H}$

$\mathrm{N} H$

$\mathrm{N} \quad \mathrm{H}$

$\mathrm{N}$ Ch

A $\mathrm{H}$

$\mathrm{N} \quad \mathrm{Cl}$ 
Ranunculus minutiflorus Bert. ex Phil.

Ranunculus stenopetalus Hook.

Ranunculus uniflorus Phil. ex Reiche

Raphanus sativus L.

Relbunium hypocarpium (L.) Hemsl.

Rhaphithamnus spinosus (A.L.Juss.) Mold.

Ribes magellanicum Poir.

Rubus geoides J.E.Sm.

Rumex conglomeratus Murr.

Rumex sanguineus L.

Sagina apetala Ard.

Salix viminalis L.

Sarmienta repens Ruiz et Pav.

Selliera radicans Cav.

Senecio acanthifolius Hombr. et Jacquinot

Senecio candidans DC.

Senecio otites Kunze et DC.

Senecio smithii DC.

Solanum coxii Phil.

Solanum nigrum L.

Sonchus asper (L.) Hill.

Sonchus oleraceus L.

Spergularia rubra (L.) J.Presl. et K.Presl.

Stachys grandidentata Lindl.

Tepualia stipularis (Hook. et Arn.) Griseb.

Ugni molinae Turcz.

Urtica dioica L.

Valeriana magna Vahl.

Weinmannia trichosperma Cav.

Liliopsida (Monocotiledóneas)

Agrostis capillaris L.

Aira caryophyllea L.

Ammophila arenaria (L.) Link.

Astelia pumila (G.Forster) R.Br.

Carex fuscula D'Urv.

Carex pseudocyperus L.

Carex pumila Thumb.

Carex riparia Curtis

Carpha alpina R.Br.

Chaetotropis imberbis (Phil.) Björk.

Chloraea magellanica Hook.f.

Chusquea montana Phil.

Cyperus eragrostis Lam.

Dactylis glomerata L.

Eleocharis pachycarpa Desv.

Fascicularia bicolor (Ruiz et Pav.) Mez.

Festuca purpurascens Banks et Soland. ex

Hook.f.

Festuca rubra L.

Gaimardia australis Gaud.
Ranunculaceae

Brassicaceae
Rubiaceae
Verbenaceae
Saxifragaceae
Rosaceae
Polygonaceae

Caryophyllaceae

Salicaceae

Gesneriaceae

Goodeniaceae

Asteraceae

Solanaceae

Asteraceae

Caryophyllaceae

Lamiaceae

Myrtaceae

Urticaceae

Valerianaceae

Cunoniaceae

Poaceae

Liliaceae

Cyperaceae

Poaceae

Orchidaceae

Poaceae

Cyperaceae

Poaceae

Cyperaceae

Bromeliaceae

Poaceae

Centrolepidaceae
Botón de oro

chico

Penchaico

Rabanito silvestre

Relbún

Huayún

Zarzaparrilla

Miñe-miñe

Romaza

Romaza marina

Sagina

Sauce mimbre

Medallita

-

Hualtata

Lampazo

amarillo

Lampazo

Tomatillo

Nilhue

Taisana

Ortiga muda

Tepú

Murta

Ortiga

Valeriana

Tineo

Chépica

Aira

Amófila

Astelia

Cortadera chica

Cortadera

Cortadera enana

Cortadera azul

Carfa

-

Orquídea

Quila enana

Cortadera

Pasto ovillo

Quilmén

Chupalla

Festuca
$\mathrm{N}$

$\mathrm{N} \mathrm{T}$

$\mathrm{N} H$

A $\mathrm{T}$

$\mathrm{N}$ Ch

$\mathrm{N} P h$

$\mathrm{N} \quad \mathrm{Ph}$

$\mathrm{N} \quad \mathrm{Cr}$

A $\mathrm{H}$

$\mathrm{N} \quad \mathrm{H}$

A $\mathrm{T}$

A $\mathrm{Ph}$

$\mathrm{N}$ Ep

$\mathrm{N}$ Ch

$\mathrm{N} \mathrm{H}$

$\mathrm{N} \mathrm{H}$

$\mathrm{N} \quad \mathrm{H}$

$\mathrm{N} \quad \mathrm{H}$

$\mathrm{N}$ T

A $\mathrm{T}$

A $\mathrm{T}$

A $\mathrm{T}$

A Ch

$\mathrm{N}$ Ch

$\mathrm{N} P h$

$\mathrm{N} P h$

A T

$\mathrm{N} H$

$\mathrm{N} \quad \mathrm{Ph}$

A $\mathrm{H}$

A $\mathrm{T}$

A $\mathrm{H}$

$\mathrm{N} \quad \mathrm{Ch}$

$\mathrm{N} \mathrm{H}$

$\mathrm{N} \quad \mathrm{H}$

$\mathrm{N} H$

$\mathrm{N} H$

$\mathrm{N} \quad \mathrm{H}$

$\mathrm{N} \quad \mathrm{H}$

$\mathrm{N} \mathrm{H}$

$\mathrm{N} \quad \mathrm{Ch}$

$\mathrm{N} H$

A $\mathrm{H}$

$\mathrm{N} H$

$\mathrm{N} \mathrm{H}$

$\mathrm{N} \quad \mathrm{H}$

A $\mathrm{H}$

$\mathrm{N} \quad \mathrm{Ch}$ 
Greigia landbeckii (Phil.) Skottsb.

Holcus lanatus L.

Juncus balticus Willd.

Juncus microcephalus Humb., Bonpl. et Kunth

Juncus pallescens Lam.

Juncus planifolius R.Br.

Lemna minima Phil.

Leymus arenarius (L.) Hochst.

Libertia chilensis (Mol.) Gunckel

Luzuriaga polyphylla (Hook.) Macbr.

Luzuriaga radicans Ruiz et Pav.

Marsippospermum grandiflorum (L.f.) Hook.f.

Oreobolus obtusangulus Gaud.

Panicum urvilleanum Kunth.

Philesia magellanica J.F.Gmel.

Phleum pratense L.

Poa alopecurus (Gaud.) Kunth.

Poa annua L.

Polypogon australis Brongn.

Ruppia filifolia (Phil.) Skottsb.

Schoenus rhynchosporoides (Steud.) Kuek.

Scirpus californicus (C.A.Mey.) Steud.

Scirpus cernuus Vahl.

Scirpus inundatus (R.Br.) Poir.

Scirpus olneyi A.Gray ex Engelm. et A.Gray

Tapeinia pumila (Hook.f.) Baillon

Tetroncium magellanicum Willd.

Triglochin concinna Davy

Uncinia erinacea (Cav.) Pers.

Uncinia phleoides (Cav.) Pers.

Uncinia tenuis Poepp. ex Kunth.
Bromeliaceae
Poaceae
Juncaceae

Lemnaceae

Poaceae

Iridaceae

Philesiaceae

Juncaceae

Cyperaceae

Poaceae

Philesiaceae

Poaceae

Ruppiaceae

Cyperaceae

Iridaceae
Juncaginaceae

Cyperaceae
Nocha

Pasto dulce

Junquillo

amarillo

Junquillo

Hierba de la vaca

Junquillo pasto

Lenteja de agua

Coirón de arena

Calle-calle

Coralito

Quilmén

-

Pasto peludo

Coicopihue

Poa

Pasto piojillo

Cola de zorro

-

Quilmén

Totora

Can-cán

Chan-chán

-

Lirio enano

-

Hierba de la

paloma

Clin-clín chico

Clin-clín
N H

A $\mathrm{H}$

$\mathrm{N} \mathrm{H}$

$\mathrm{N} \quad \mathrm{H}$

$\mathrm{N} H$

$\mathrm{N}$ T

$\mathrm{N} \mathrm{Cr}$

A $\mathrm{H}$

$\mathrm{N} \quad \mathrm{H}$

$\mathrm{N} \quad \mathrm{Cl}$

$\mathrm{N}$ Ch

$\mathrm{N} \mathrm{H}$

$\mathrm{N} \quad \mathrm{Ch}$

$\mathrm{N} \mathrm{H}$

$\mathrm{N} \quad \mathrm{Ph}$

A $\mathrm{H}$

$\mathrm{N} \mathrm{H}$

A $\mathrm{T}$

$\mathrm{N} \quad \mathrm{H}$

$\mathrm{N}$ Cr

$\mathrm{N} \mathrm{H}$

$\mathrm{N}$ Cr

$\mathrm{N}$ Cr

$\mathrm{N} \quad \mathrm{Cr}$

$\mathrm{N} H$

$\mathrm{N}$ Ch

$\mathrm{N} \mathrm{H}$

$\mathrm{N} \quad \mathrm{H}$

$\mathrm{N} \quad \mathrm{H}$

$\mathrm{N} H$

$\mathrm{N} \mathrm{H}$

La ausencia de familia y nombre común indica que vale el inmediatamente superior.

Origen fitogeográfico (O): $\mathrm{N}$ - nativo, $\mathrm{A}$ - adventivo. Formas de vida (FV): $\mathrm{Ph}$ - fanerófitos,

Ep - epífitos,

$\mathrm{Cl}$ - trepadores, $\mathrm{Ch}$ - caméfitos, $\mathrm{H}$ - hemicriptófitos, $\mathrm{Cr}$ - criptófitos, T - terófitos.

Anexo 2. Lista de sinónimos usados en este trabajo.

\begin{tabular}{ll}
\multicolumn{1}{c}{ Sinónimo } & \multicolumn{1}{c}{ Nombre válido } \\
\hline Callitriche verna L. & Callitriche palustris L. \\
Cerastium fontanum Baumg. & Cerastium holosteoides Fries \\
Chusquea nigricans Phil. & Chusquea montana Phil. \\
locenes acanthifolius (Hombr. et Jacquinot) B.Nord. & Senecio acanthifolius Hombr. et Jacquinot \\
Juncus arcticus Willd. & Juncus balticus Willd. \\
Juncus dombeyanus J.Gay ex Lah. & Juncus pallescens Lam. \\
Lathyrus maritimus Big. & Lathyrus japonicus Willd. \\
Laureliopsis philippiana (Looser) Schodde & Laurelia philippiana Looser \\
Lemna minuscula Herter & Lemna minima Phil. \\
Megalastrum spectabile (Kaulf.) A.R.Sm. et & Ctenitis spectabilis (Kaulf.) Kunkel \\
R.C.Moran & Lythrum portula L. \\
Peplis portula L. & Plantago australis Lam. \\
Plantago tomentosa Lam. &
\end{tabular}




\section{LITERATURA CITADA}

Amigo, J. \& C. Ramírez 1998. A bioclimatic classification of Chile: woodland communities in the temperate zone. Plant Ecology 136: 9-26.

Amigo, J., C. Ramírez \& L. G. Quintanilla 2004. The Nothofagus nitida (Phil.) Krasser woodlands of southern Chile in the northern half of their range: phytosociological position. Acta Botanica Gallica 151: 3-31.

Amigo, J., C. Ramírez \& L. G. Quintanilla 2007. Mantle communities of the temperate woodlands of South Central Chile: a phytosociological study of the order Aristotelietalia chilensis. Phytocoenologia 37: 269-319.

Castro, V. \& M. Romo 2006. Tradiciones culturales y biodiversidad.. En: Saball, P., Arroyo, M., Castilla, J.C., Estades, C., Ladrón de Guevara, J.M., Larraín, S., Moreno, C., Rivas, F., Rovira, J., Sánchez, A., Sierralta, L. (eds.). Biodiversidad de Chile. Patrimonio y Desafíos. Comisión Nacional del Medio Ambiente, Santiago, Chile. pp. 468-493.

Darwin, C. R. 1845. Journal of researches into the natural history and geology of the countries visited during the voyage of H.M.S. Beagle round the world. John Murray, London. 519 pp.

Dierschke, H. 1994. Pflanzensoziologie. Eugen Ulmer, Stuttgart. 683 pp.

Ellenberg, H \& D. Mueller-Dombois 1966. A key to Raunkaier plant life forms with revised subdivisions. Bericht Geobotanisches Institut ETH, Stiftung Rübel Zürich 37: 56-73.

Eskuche, U. G. 2005. Las comunidades vegetales litorales de los lagos patagónicos y de los canales magallánicos. Folia Botanica et Geobotanica Correntesiana 18: 1-31.

Fuenzalida, H. 1965. Clima. En: Geografía Económica de Chile. CORFO, Fundación Pedro Aguirre Cerda, Santiago. pp: 99-152.

Fuenzalida, R. 1978. Nuevos antecedentes de cambios de morfología costera después de los sismos de 1960, Archipiélago de Los Chonos. Trapananda 1: 71-75.

Gajardo, R. 1994. La vegetación natural de Chile, Clasificación y distribución geográfica. Editorial Universitaria, CONAF, Santiago. $165 \mathrm{pp}$

Hajek, E. R. \& F. di Castri 1975. Bioclimatografía de Chile. Ediciones Universidad Católica de Chile, Santiago. 114 pp.

Hauenstein, E., C. Ramírez, M. Latsague \& D. Contreras 1988. Origen fitogeográfico y espectro biológico como medida del grado de intervención antrópica en comunidades vegetales. Medio Ambiente 9: 140-142.

Hervé, F. \& C. M. Fanning 2001. Late Triassic detrital zircons in meta-turbidites of the Chonos $\mathrm{Me}$ tamorphic Complex, southern Chile. Revista Geológica de Chile 28: 91-104.

Heywood, V.H. 1982. Blütenpflanzen der Welt. Birkhäuser Verlag, Basel, Boston, Stuttgart. 336 pp.

Hildebrand-Vogel, R. 1988. Ersatzgesellschaften des nordwestpatagonischen Lorbeerwaldes in Chile zwischen 42 und $45^{\circ}$ s. Br. Flora 180: 161-176.

Jaccard, P. 1912. The distribution of the flora in the Alpine zone. New Phytologist 11: 37-50.

Jurasinski, G. 2007. Simba: a collection of functions for similarity calculation of binary data. URL: cran.r-project.org/package $=$ simba.

Knapp, R. 1984. Sampling methods and taxon análisis in vegetation sciences. Dr.Junk Publishers, La Haya, Boston, Lancaster. 370 pp.

Luebert, F. \& P. Pliscoff 2006. Sinópsis bioclimática y vegetacional de Chile. Editorial Universitaria, Santiago. 316 pp.

Manríquez, V. 2004. Las poblaciones indígenas en Chile en el siglo XVI. En: F. Rothhammer \& E. Llop (eds.). Poblaciones chilenas cuatro décadas de investigaciones bioantropológicas. Editorial Universitaria, Santiago. Pp: 19-36.

Marticorena, C. \& M. Quezada 1985. Catálogo de la flora vascular de Chile. Gayana Botanica 42: 5-161.

Marticorena, C. \& R. Rodríguez 1995-2003. Flora de Chile. Universidad de Concepción. 2 vols.

Mena, F. 1985. Presencia indígena en el litoral de Aisén. Trapananda 8: 203-213.

Oberdorfer, E. 1960. Pflanzensoziologische Studien in Chile, ein Vergleich mit Europa. J. Cramer, Weinheim. 208 pp.

Oksanen, J., R. Kindt, P. Legendre, B. O'Hara, G. L. Simpson, P. Solymos, M. Henry, H. Stevens \& H. Wagner 2009. Vegan: community ecology package. URL: cran.r-project.org/ package=vegan. 
Peralta, M. \& C. Homann. 1978. Suelos de las islas Guaytecas y de la isla Concoto en Los Chonos. Ciencias Forestales 1: 29-40.

Pisano, E. 1981. Bosque fitogeográfico de FuegoPatagonia. Anales Instituto Patagonia (Chile) 12: $159-171$.

Plafker, G. 1972. The Alaska earthquake of 1964 and Chilean earthquake of 1960: implications for arc tectonics. Journal of Geophysical Research 77: 901-925.

Quintanilla, V. 1981. Carta de las formaciones vegetales de Chile. Contribuciones Científicas y Tecnológicas, Area geociencias VII USACH Santiago 47: 5-32.

Quintanilla, V. 1989. Fitogeografía y cartografía vegetal de Chile Austral. Contribuciones Científicas y Tecnológicas, Area geociencias VII USACH Santiago 87: 5-27.

R Development Core Team 2009. R: a language and environment for statistical computing. $\mathrm{R}$ Foundation for Statistical Computing, Vienna. URL: cran.r-project.org.

Ramírez, C. \& M. Romero 1978. El Pacífico como agente diseminante en el litoral chileno. Ecología (Argentina) 3: 19-30.

Ramírez, C. \& H. Figueroa 1985. Delimitación ecosociológica del bosque valdiviano (Chile) mediante análisis estadísticos multivariados. Studia Oecologica 6: 105-124.

Ramírez, C., C. San Martín, D. Contreras \& J. San Martín 1989. Flora de las marismas del centrosur de Chile. Medio Ambiente 10: 11-24.

Ramírez, C., V. Finot, C. San Martín \& A. Ellies 1991. El valor indicador ecológico de las malezas del Centro-Sur de Chile. Agro Sur 19: 94-116.

Ramírez, C. \& C. San Martín 1993. La transformación antrópica de la vegetación de los ñadis del área Mapuche en el Centro-Sur de Chile. Boletin Museo Regional de la Araucanía 4: $205-214$.

Ramírez, C., M. Álvarez \& A. Díaz 2004. Resultados botánicos de la primera expedición científica a la Isla Guamblin (Archipiélago de los Chonos, XI Región, Chile). Revista Geográfica de Valparaíso 35: 225-242.

Ramírez, C. \& C. San Martín 2006. Ecosistemas dulceacuícolas. En: P. Saball, M. Arroyo, J.C. Castilla, C. Estades, J.M. Ladrón de Guevara,
S. Larrain, C. Moreno, F. Rivas, J. Rovira, A. Sánchez, L. Sierralta (eds.). Biodiversidad de Chile. Patrimonio y Desafíos. Comisión Nacional del medio Ambiente, Santiago, Chile. Pp. 112-124.

Ramírez, C., M. Álvarez , A. Díaz \& G. Toledo 2006a. Biodiversidad vegetal de la isla Ipún en la Reserva Nacional Las Guaitecas (Comuna de Cisnes, XI Región, Chile). Revista Geográfica de Valparaíso 37: 45-66.

Ramírez, C., M. Álvarez, A. Díaz \& G. Toledo 2006b. Estudio florístico y vegetacional de la Isla Kent (Archipiélago de Los Chonos, Reserva Nacional Las Guaitecas, XI Región, Chile). Revista Geográfica de Valparaíso 38: 55-68. Ramírez, C., M. Álvarez, A. Díaz \& G. Toledo 2007. Diversidad florística y vegetacional de la Isla Stokes (Reserva Nacional Las Guaitecas, XI Región, Chile). Revista Geográfica de Valparaíso 39: 99-111.

Reiche, K. 1896-1911. Flora de Chile. Imprenta Cervantes, Santiago. 6 vols.

Reiche, K. 1934. Geografía botánica de Chile. Imprenta Universitaria, Santiago. 423 pp.

Roig, F. A., O. Dollenz \& E. Méndez 1985. La vegetación el los canales. En: O. Boelcke, D. M. Moore \& F. A. Roig (eds.). Transecta botánica de la Patagonia Austral. Consejo Nacional de Investigaciones Científicas y Técnicas (Argentina), Buenos Aires. pp: 457-519.

Roig, F. A. 1998. La vegetación de la Patagonia. CONICET, Mendoza. 166 pp.

Rojas, G. 2002. Reyes sobre la tierra. Editorial Biblioteca Americana, Universidad Andrés Bello, Santiago. 219 pp.

Roqueferre, M. 1987. Isla Guamblin, un parque escondido. Mundo Diners Club 57: 34-38.

Sáiz, F. 1980. Experiencias en el uso de criterios de similitud en el estudio de comunidades. Archivos de Biología y Medicina Experimental 13: 387-402.

San Martín, J., C. Ramírez \& C. San Martín 1992. La flora de las dunas chilenas y sus adaptaciones morfológicas. Bosque 13: 29-39.

San Martín, C., C. Ramírez \& M. Alvarez 2003. Macrófitos como bioindicadores: Una propuesta metodológica para caracterizar ambientes dulciacuícolas. Revista Geográfica de Valparaíso 34: 243-253. 
San Martín, C., C. Ramírez \& M. Álvarez 2004. Estudio de la vegetación de "Mallines" y "Campañas" en la Cordillera Pelada (Valdivia, Chile). Revista Geográfica de Valparaíso 35: 261-273.

Scherhag, R. 1969. Klimatologie. Georg Westermann, Braunschweig. 165 pp.

Schrödl, M. \& J. Grau 2006. Nudibranchia from the remote southern Chilean Guamblin and Ipún islands (Chonos Archipelago, 44-45 S), with re-description of Rostanga pulchra MacFarland, 1905. Revista Chilena de Historia Natural 79: 3-12.

Szeicz, J., S. Haberle \& K. Bennett 2003. Dynamics of North Patagonian rainforest fine-resolution pollen, charcoal and tree-ring análisis, Chonos Archipelago, Southern Chile. Austral Ecology 28: 413-422.

Tichý, L. 2002. JUICE, software for vegetation classification. Journal of Vegetation Science 13: 451-453.

Ugent, D., T. Dillehay \& C. Ramírez 1987. Potato remains from a late pleistocene settlement in
South central Chile. Economic Botany 41: 17-27. Valenzuela, J. A. \& J. Grau 2005. Ocurrence of American mink on the Chonos Archipelago of southern Chile. Oryx 39: 15.

Veblen, T. T. \& D. H. Ashton 1978. Catastrophic influence on the vegetation of the Valdivian Andes, Chile. Vegetatio 36: 149-167.

Villagra, J., D. Montenegro, C. San Martín, C. Ramírez \& I. Álvarez 2009. Estudio de la flora liquénica de las turberas de la comuna de Tortel (Región de Aisén), Patagonia chilena. Anales Instituto Patagonia (Chile) 37(1): 53-62.

Wardle, P., C. Ezcurra, C. Ramírez \& S. Wagstaff 2001. Comparison of the flora and vegetation of the southern Andes and New Zealand. New Zealand Journal of Botany 39: 69-108.

Walter, H. 1997. Zonas de vegetación y clima. Ediciones Omega, Barcelona. 245 pp.

Westermeier, R. \& C. Ramírez 1979. Artendiversität und Nekromasse der Algen im Strandanwurf von Niebla (Valdivia, Chile). Botanica Marina 22: 241-248. 